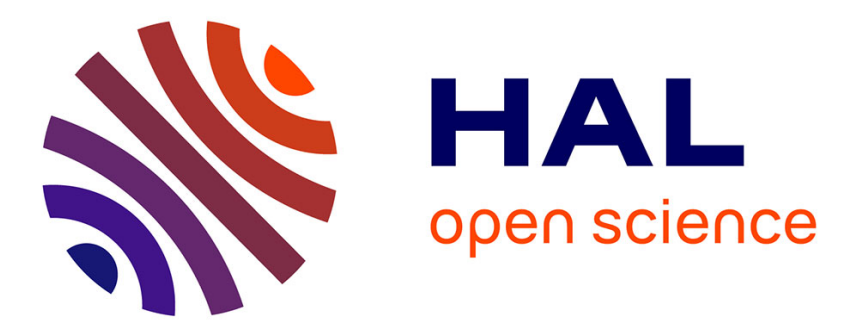

\title{
Dynamic Echo-Planar MR Imaging of the Diaphragm for a 3D Dynamic Analysis
}

Stéphane Craighero, Emmanuel Promayon, Pierre Baconnier, Jean-François Lebas, Max Coulomb

\section{- To cite this version:}

Stéphane Craighero, Emmanuel Promayon, Pierre Baconnier, Jean-François Lebas, Max Coulomb. Dynamic Echo-Planar MR Imaging of the Diaphragm for a 3D Dynamic Analysis. European Radiology, 2005, 15 (4), pp.742-748. 10.1007/s00330-004-2482-2 . hal-00306711

\section{HAL Id: hal-00306711 https://hal.science/hal-00306711}

Submitted on 28 Jul 2008

HAL is a multi-disciplinary open access archive for the deposit and dissemination of scientific research documents, whether they are published or not. The documents may come from teaching and research institutions in France or abroad, or from public or private research centers.
L'archive ouverte pluridisciplinaire HAL, est destinée au dépôt et à la diffusion de documents scientifiques de niveau recherche, publiés ou non, émanant des établissements d'enseignement et de recherche français ou étrangers, des laboratoires publics ou privés. 


\title{
Dynamic Echo-planar MR Imaging of the Diaphragm for a 3D Dynamic Analysis
}

\author{
S. Craighero' ${ }^{\text {, MD, E. Promayon }}{ }^{2}$, Ph.D., P. Baconnier², Ph.D., \\ JF. Lebas 3 , MD, M. Coulomb ${ }^{1}$, MD
}

1 From the department of Radiology, Hôpital Michallon, 38700 La Tronche Grenoble, France, Phone: +33/0-4 767652 41, Fax: +33/0-4 76765901

2 TIMC-IMAG CNRS UMR 5525, Université J. Fourier, Institut d'Ingénierie de l'Information de Santé, Faculté de Médecine, 38706 La Tronche Grenoble, France, Phone: +33/0-4 5652 00 03, Fax: +33/0-4 56520055

${ }^{3}$ From the Unity of MRI, Hôpital Michallon, 38700 La Tronche Grenoble, France, Phone: +33/0-4 76765491

(email: Emmanuel.Promayon@imag.fr) 


\section{ABSTRACT}

Objective: To prove the feasibility of 3D reconstructions of the diaphragm during respiratory cycle, using EPI sequences.

Materials and Methods: EPI acquisition, $270 \mathrm{~ms} / \mathrm{image}$, on a healthy subject breathing spontaneously and at $0.1 \mathrm{~Hz}$. Continuously recorded respiratory signal allowed for retrospective synchronization with respiratory phases for reconstruction of successive diaphragm surfaces using a specifically designed software. Displacements, area and volume changes of the diaphragm were quantified.

Results: Our measurements were comparable with literature data. Reconstructed surfaces allowed in vivo diaphragm dynamics evaluation, in terms of displacements, area and volume variations.

Conclusion: EPI has adequate spatial and temporal resolution for studying diaphragm dynamics during natural breathing.

\section{KEYWORDS}

Diaphragm, 3-D imaging, image reconstruction, Dynamic EPI. 


\section{INTRODUCTION}

There is only limited information in the literature on the in vivo three-dimensional structure of the human diaphragm. Previous studies were restricted to various lung volumes, during breath holding and, therefore, not adequate for the study of patients with Chronic Obstructive Pulmonary Disease (COPD) [1-6].

Recent techniques for subsecond scan acquisitions, such as Echo-Planar Imaging (EPI), allow multislice dynamic MR studies and improve previous fast gradient recalled echo MR studies [1,3,4,7-10].

Previous studies have suggested the possibility of using MR imaging to investigate the effects of thoracic structural changes on the pulmonary function that result from pulmonary resection, transplantation, or disease [4]. In these cases, dynamic EPI with 3D dynamic reconstruction appeared to be a promising approach to study diaphragmatic function.

Our aim was to prove the feasibility of 3D dynamic reconstruction of the diaphragm during the respiratory cycle, using dynamic EPI on a healthy subject. In addition, we provide details on the computer assisted method used to model the $3 \mathrm{D}$ diaphragmatic surfaces and to display a 3D animation of the respiratory cycle.

\section{METHODS}

\section{Subject}

We studied a healthy male, aged 50, $188 \mathrm{~cm}$ tall, weighting $92 \mathrm{~kg}$, in a supine position. This subject is a respiratory physiologist used to control his breathing for experimental recordings. His informed consent was obtained after the nature of the procedure had been fully explained. 


\section{MRI Technique}

Multislice dynamic EPI were performed at 1.5 T (Philips Gyroscan).

The imaging sequence was a Fast Field Echo-Echo Planar Imaging (FFE-EPI), with the following operating conditions: body coil, repetition time of 0.113 seconds, echo time of 0.040 seconds, flip angle of $20^{\circ}, 128$ phase-encoding steps, intersection gap of 10 $\mathrm{mm}$ (sagittal) and $1.5 \mathrm{~mm}$ (coronal), section thickness of $10 \mathrm{~mm}$, field of view of 45.0 $\mathrm{cm}$. With this parameter settings, each slice acquisition lasted about 0.270 seconds.

\section{Data Acquisition}

This study was performed with two different breathing conditions: "quiet breathing" and "maximal amplitude breathing", as defined below. Our multislice dynamic MR studies consisted in 4 multislice dynamic acquisitions. Each of them consisted in 45 series of 15 slices, acquired during 3 minutes. The coronal slices were positioned from a costovertebral plane to a sternal plane, and the sagittal slices from the right lateral thoracic wall to the left lateral thoracic wall. Hence one multislice dynamic acquisition was performed for each plane (coronal and sagittal), and for each type of breathing ("quiet breathing" and "maximal amplitude breathing").

The total number of images acquired for each type of breathing was $45 \times 15 \times 2=1350$ images. The BIOPAC MP100 system (BIOPAC Systems Inc., Santa Barbara, California) connected with a respiration transducer made of a nylon belt and silicon rubber strain gauge allowed continuous recording of thoracic and abdominal circumference variations on a laptop computer (see figure 1).

Within each multislice dynamic acquisition, we selected the "best" minute, i.e. when the respiratory cycles were the most reproducible as evaluated on thoracic and abdominal circumference variations. Finally, we extracted $15 \times 15 \times 2=450$ images (in sagittal and coronal planes) for each type of breathing. 
To ensure breathing consistency among dynamic acquisitions, programmed light flashes triggered each inspiration and expiration. The subject had to synchronize his breathing with the light signals. For "quiet breathing", inspiratory and expiratory duration were respectively set at 2 seconds and 3 seconds, mimicking our subject's spontaneous values observed during a preliminary respiratory recording. Therefore the subject's respiratory cycles were synchronized with his own spontaneous rhythm. For "maximal amplitude breathing", the subject was asked to continuously complete maximal volume amplitudes in 5 seconds inspiration and 5 seconds expiration.

An external marker was fixed around the costal margin to identify the attachment of the diaphragm. This marker was a flexible tube filled with water, taped to the skin, as previously described by Gauthier et al. [2].

\section{Post-synchronization}

The respiratory cycle was divided into 10 intervals of time of the same duration, named "phases". By convention, the respiratory cycle begins at phase 1 (beginning of inspiration). The "quiet breathing" cycle was divided in 4 inspiratory phases followed by 6 expiratory phases, each phase lasting 0.5 seconds; while the "maximal amplitude breathing" cycle was divided in 5 inspiratory phases followed by 5 expiratory phases, each phase lasting 1 second.

By synchronizing the MRI acquisition with the respiratory signal, we have been able to determine in which respiratory phase each image was acquired. Hence, each slice was located by specific spatial and time coordinates. We then defined a "3D reconstructed phase" (3D-RP) as a reconstructed MRI volume of the diaphragm for a given respiratory phase. A 3D-RP is made of 15 sagittal and 15 coronal images, where each image was acquired during this given respiratory phase, see figure 1 for details. This step was called post-synchronization. At the end of post-synchronization, 10 3D-RP for each type 
of breathing were created, i.e. 300 images for each type of breathing. Remaining images of the "best" minute were kept to allow later replacement if needed.

\section{Surface segmentation}

We designed a software to display the $3 \mathrm{D}-\mathrm{RP}$ in a $3 \mathrm{D}$ interactive environment. The software took into account the MRI operating condition data: sagittal and coronal shift, gap and thickness of the slices. The user was free to move in the 3D environment and to display any slice of a 3D-RP. An initial 3D flat surface mesh was then automatically generated. Each node of this initial surface mesh was at the intersection of a sagittal and a coronal plane. Thus, the initial surface mesh was composed of $15 \times 15$ nodes.

The diaphragm contour was then directly segmented by manually deforming the initial surface mesh (see figure 2). To deform the mesh, the user was allowed to move any node or group of nodes in the 3D environment but displacements were constrained on the slice intersections. The software also allowed the radiologist to add or delete new nodes when needed (e.g. in the apposition zone). As the segmentation was directly carried out in $3 \mathrm{D}$, no ulterior reconstruction processes were needed. The use of sagittal and coronal slices was made depending on the considered diaphragm part. Sagittal slices were used for the segmentation of the posterior zone of apposition, and coronal slices for the lateral zone of apposition. For all the other parts, the sagittal and coronal sets of images were both used, each mesh node being precisely adjusted by an alternative visualization of both slice directions.

If at that stage the diaphragm contour at the intersection of one sagittal and one coronal image differed from more than one pixel, the image offering the worst coherence and continuity with its surrounding was discarded and replaced by another image taken amongst the ones available for the same phase and spatial position. The caudal boundary of the diaphragm was defined by the position of the external marker. As the 
diaphragmatic crura could not be distinguished from the spine or aorta, anatomical references were considered to obtain the lumbar attachment of the diaphragm.

Finally, $103 \mathrm{D}$ meshes corresponding to the diaphragmatic surfaces were obtained (see figure 3). The diaphragmatic surface was composed of 289 nodes. Mean distance between adjacent nodes was $2 \mathrm{~cm}$ in the sagittal plane and $1.15 \mathrm{~cm}$ in the coronal plane. The software also allowed us to build animations of these 3D diaphragmatic surfaces showing all the diaphragmatic deformations during the respiratory cycle.

\section{Landmarks}

From the 3D surface at the end of expiration, we chose the left and right apexes (respectively called LA and RA) by picking the highest nodes on each hemidiaphragm. Four coronal planes were chosen: the "left apex" plane (containing LA), the "right apex" plane (containing RA), the "anterior" plane (halfway between the RA plane and the anterior costophrenic angle) and the "posterior" plane (halfway between the RA plane and the posterior costophrenic angle), similarly to [8].

\section{Area and volume measurement}

All the 3D surface mesh nodes were automatically linked by triangular facets. Therefore a 3D surface mesh described a 3D diaphragmatic surface (see figure 4). The distance data and areas from the 3D diaphragmatic surfaces could be then automatically obtained. The total diaphragm area was approximated by the sum of all the triangular facet areas. We defined the "volume under diaphragm" as the sum of the volumes of all the tetrahedron defined by each facet and the origin of a fixed Cartesian coordinate system (see figure 4). Therefore only variations of this volume were relevant and studied.

The diaphragmatic area was computed in $\mathrm{cm}^{2}$ and the "volume under diaphragm" in $\mathrm{cm}^{3}$. 


\section{RESULTS}

\section{Image quality}

Motion artifacts from diaphragmatic movement or cardiac pulsation did not interfere with the diaphragm visibility. In some images, a thin, black line separating the pericardium from the abdominal fat was observed.

\section{General observations}

We noted that LA and RA were exactly positioned on 2 sagittal paramedian slices at +8 $\mathrm{cm}$ and $-8 \mathrm{~cm}$ from the thorax middle. LA was $2.2 \mathrm{~cm}$ posterior to RA. The maximal excursion was $3.85 \mathrm{~cm}$ for RA and $4.85 \mathrm{~cm}$ for LA.

The sets of nodes of the 4 coronal planes (from front to back: anterior, RA, LA and posterior) were plotted on figure 5 for phase 1 and 5 (beginning and end of inspiration, maximal amplitude breathing). The mean excursion of all these nodes was also computed (see table 1).

\section{Area measurement}

Area variations during the cycle were plotted in figure 6 for both respiratory maneuvers. The comparison between the 2 breathing conditions showed that the diaphragmatic area was minimal during phase 5 in "quiet breathing" and phase 6 in "maximal amplitude breathing". This was explained by the 2 types of respiratory synchronization. During "quiet breathing", this area changed from a minimum of $1,431.5 \mathrm{~cm}^{2}$ (phase 5) to a maximum of $1,537.8 \mathrm{~cm}^{2}$ (phase 10), a variation of $106.3 \mathrm{~cm}^{2}$. During "maximal amplitude breathing", this area changed from a minimum of $1,158.0 \mathrm{~cm}^{2}$ (phase 6) to a maximum of $1,538.4 \mathrm{~cm}^{2}$ (phase 10), a variation of $380.4 \mathrm{~cm}^{2}$. The maximal increase of the diaphragm surface was observed between phase 7 and 8 in "quiet breathing", and phase 8 and 9 in "maximal amplitude breathing", representing respectively $46.4 \%$ and $51.3 \%$ of the total variation. 


\section{Volume measurement}

The volume variations during the cycle were plotted in figure 7 for both respiratory maneuvers. The minimal values were observed during phase 5 in "quiet breathing" and phase 6 in "maximal amplitude breathing".

During "quiet breathing", the "volume under diaphragm" variation was $562.8 \mathrm{~cm}^{3}$ from phase 5 to phase 10. During "maximal amplitude breathing", this variation was 2,038.9 $\mathrm{cm}^{3}$ from phase 6 to phase 10 . The maximal decrease of the volume was observed between phase 7 and 8 in "quiet breathing", and phase 8 and 9 in "maximal amplitude breathing", representing respectively $47.0 \%$ and $51.7 \%$ of the total expiration volume variation.

We also studied all the nodes representing the attachment of the diaphragm. Their displacements were mainly on the anterior part and sagittal. The maximal displacement observed was $0.5 \mathrm{~cm}$ for "quiet breathing", and $1 \mathrm{~cm}$ for "maximal amplitude breathing". Volume and surface variations were compared by plotting a XY graph, showing a nearly linear relationship between these values (figure 8).

\section{DISCUSSION}

\section{MR imaging}

EPI sequences for studies on diaphragmatic motion were advocated by previous works of Gierada et al. and Kanematsu et al. [8, 9]. It was suggested that the diaphragmatic surface and its motion during breath movements could thus be studied simultaneously. Moreover it was plausible to foresee that respiratory constraints for the subject as well as entry-section artifacts, and cardiac or respiratory motion artifacts could be decreased using EPI. In our protocol, we reached a time resolution of 0.270 seconds per slice which is superior to the 10 seconds obtained with spin echo MRI [2,5] and 1.2 seconds obtained for fast gradient recalled echo MRI [1,3,4,7-10]. Recently, the acquisition rate 
of fast gradient recalled echo has been increased, acquiring 22-27 coronal slices and 3033 sagittal slices during 22-33 seconds of apnea for 5 healthy subjects at three different pulmonary volumes [1].

In our study, the total acquisition lasted 3 minutes, but we noted $a$ posteriori that a shorter acquisition of 1 minute would have been enough to reconstruct the 10 diaphragmatic surfaces.

Concerning the $2 \mathrm{D}$ resolution, our $128 \times 128$ matrix ( $3.5 \mathrm{~mm}$ pixels) was of reasonable quality compared to similar studies where a $128 \times 256$ matrix was used $[2-5,8,9]$. This resolution was enough to adequately segment the diaphragmatic surfaces.

We used 15 sagittal slices as previous studies [2], but our acquisition protocol allowed us to arbitrarily choose this number without adding any constraints for the subject. The combination with the 15 coronal slices allowed us to over-sample the diaphragmatic surfaces in the zone of apposition.

Due to the short slice acquisition time of the FFE-EPI technique, the subject did not have to hold his breath, unlike in previous studies [1-5]. Moreover, in quiet breathing situation, the subject breathed with minimal effort as the synchronization flash rhythm mimicked his own respiratory rhythm. Thus, this experimental protocol could be easier to apply to patient studies than previously proposed protocols, particularly in chronic obstructive and restrictive pulmonary diseases.

Using fast gradient recalled echo sequence [8], previous investigations observed an increase in the MRI signal intensity attributed to the movements of the thoracic wall and of the lung, which, when included in the slice, produced an entry-section phenomenon, similar to the one caused by blood flow. In our study, we had no entry-section artifact. This could be a consequence of the short slice acquisition time during which the wall movement could only be limited. The thin, black line separating the pericardium from the abdominal fat observed in some images was probably due to chemical shift artifact. 


\section{Quantitative analysis}

LA was $2.2 \mathrm{~cm}$ posterior to RA, as observed in [8]. The maximal apex excursions during "maximum amplitude breathing" were $3.85 \mathrm{~cm}$ for RA and $4.85 \mathrm{~cm}$ for LA. This difference could be explained by the fact that LA was $2.2 \mathrm{~cm}$ posterior to RA and that the craniocaudal displacement of the diaphragm was more important for the posterior part $(4.14 \mathrm{~cm})$ than for the anterior part $(1.64 \mathrm{~cm})$, as also observed in [8]. Our measures were close to those presented in previous works [8]: $4.2 \mathrm{~cm}$ on the right hemidiaphragm and $4.4 \mathrm{~cm}$ for the left hemidiaphragm. Note that the latter was observed for Total Lung Capacity (TLC) maneuvers.

Paiva et al [5] measured a mean diaphragmatic surface of $1,151 \mathrm{~cm}^{2}$ by using a 3D reconstruction from MRI data acquired on 4 subjects in apnea at Functional Residual Capacity (FRC). Gauthier et al [2] measured the diaphragmatic surface at 4 different lung volumes on 4 subjects: 1,461 $\mathrm{cm}^{2}$ at Residual Volume (RV), 1,351 $\mathrm{cm}^{2}$ at FRC, $1,130 \mathrm{~cm}^{2}$ at $\mathrm{FRC}+1 / 2$ inspiration and $925 \mathrm{~cm}^{2}$ at TLC. Using CT scan imaging, Pettiaux et al found similar results [6]. Cluzel et al [1] calculated a smaller diaphragmatic surface area: $1,128 \mathrm{~cm}^{2}$ at RV, $997 \mathrm{~cm}^{2}$ at FRC, and $584 \mathrm{~cm}^{2}$ at TLC. We found a diaphragmatic surface of $1,537.8 \mathrm{~cm}^{2}$ at the end of expiration (close to RV) and $1,158 \mathrm{~cm}^{2}$ at the end of inspiration (close to TLC). This values are comparable to the values given in [2] (subjects mean height $1.79 \mathrm{~m}$ and mean weight $70 \mathrm{~kg}$ ) considering the body weight influences [11].

The "volume under diaphragm" values were close to the classical reported respiratory physiology values (approximately 0.5 liter in "quiet breathing " and 2 liters in "maximal amplitude breathing"). They resulted in a ventilation of 6 liters/min for "quiet breathing" corresponding to a normal quiet breathing, and 12 liters/min for "maximal amplitude breathing" corresponding to a voluntary hyperventilation. Cluzel et al [1] estimated the volume under the diaphragmatic dome at $\operatorname{RV}\left(3,228 \mathrm{~cm}^{3}\right)$ and at TLC $\left(1,054 \mathrm{~cm}^{3}\right)$. Its 
variation $\left(2,174 \mathrm{~cm}^{3}\right)$ is close to our values obtained during "maximal amplitude breathing", a maneuver which does not reach RV and TLC. The size of our rather tall and heavy subject $(1.88 \mathrm{~m}, 92 \mathrm{~kg})$ probably explains that his variation during "maximal amplitude breathing" is comparable to the corresponding variation obtained during TLC maneuvers in the "normal" subjects of study [1] (mean height $1.80 \mathrm{~m}$, mean weight 79 $\mathrm{kg})$.

\section{Limitations}

The flexible tube used as an external marker of costal attachments was fixed on the skin, meaning that it was at a distance of approximately $1 \mathrm{~cm}$ from the true costal attachments [2]. This might have introduced an error in the tracking of the rib movement. The flexible tube could also be difficult to place on an obese subject. Another limitation could arise when a reproducible respiratory pattern is required from untrained or ill subjects. Nevertheless, asking a patient to breath synchronously with a light signal mimicking his spontaneous rhythm is probably less constraining than asking for voluntary long apneas.

The "maximal amplitude breathing" corresponded to a maximal inspiration and maximal expiration of 5 seconds each. Using this technique, the subject could not perform a real "Total Lung Capacity" maneuver, nor "Residual Volume" expiration maneuver.

The nodes located on the diaphragmatic attachments (the base of the cone defined as the "volume under diaphragm", see figure 4) did not migrate much. Therefore, the "volume under diaphragm" variations were principally due to the diaphragmatic cupola deformation. We think that the "volume under diaphragm" variation observed during the respiration gave an approximation of the pulmonary volume variation due to the diaphragmatic action. But this has to be compared with plethysmographic recordings $[1$, 4]. 
The surface segmentation step of our method, even if facilitated by the use of our software, was still time consuming (approximatively $1 \mathrm{~h} 30$ for the first surface reconstruction). However, the segmentation step was facilitated by starting from the first reconstructed surface. The radiologist only had to adjust the nodes (approximatively 30 minutes for each following surface reconstruction). The further automation of this step should be very helpful but is a real challenge for segmentation algorithms. Because of the MRI properties (e.g. the chemical shift artifacts) and the diaphragm morphology, the zone of apposition, crura, and more generally all frontiers are extremely difficult to detect with an automatic algorithm. A semi-automatic algorithm could select the nearest diaphragmatic surface among a model database and make the initial global adjustment.

\section{Practical Applications and Perspectives}

The analysis of dynamic data during a respiratory cycle, using our software, allowed a functional approach of the diaphragmatic muscle. Our goal was to provide a tool, modeling the normal function of this muscle, and its role in respiratory mechanics. This model can be used in fundamental respiratory physiology and in pathology, to diagnose and assess diaphragmatic disorders. Indeed, our software was able to quantitatively evaluate diaphragmatic efficiency, by measuring both craniocaudal displacement and volume variations due to the diaphragm, as well as by visualizing its contraction kinetics and its deformation (flattening, hernia...). Dynamic MRI was also used to diagnose diaphragmatic rupture, showing paradoxical movement of the hemidiaphragm [7], or to evaluate bronchogenic carcinoma invasion of the chest wall [10].

Our current objectives are to apply our method to study Chronic Obstructive Pulmonary Disorder (COPD) with thoracic distension and diaphragmatic paralysis, diaphragmatic fatigue with ventilatory respiratory failure and neuromuscular disease. Finally, this 3D dynamic reconstruction could be integrated in a dynamic model of the trunk [12], taking 
thoracic variations [13] and lung volume modifications [1,4] into account. This dynamic model of the trunk would allow us to simulate the diaphragm function and the respiratory system for patients with emphysema before and after lung volume reduction surgery.

In conclusion, we have shown that EPI has adequate spatial and temporal resolution for studying the dynamics of the diaphragm during unconstrained physiologic breathing. The diaphragmatic surfaces can be studied individually (in terms of morphology, surfaces and volumes) or dynamically (in terms of displacement, surface and volume variations). 


\section{Acknowledgements}

The authors wish to affectionately thank Niamh Clarke and Matthieu Chabanas for carefully proofreading this paper.

This work was partly supported by the European Commission - BIOMED II programme (biomedical technology research project "BREATH" - Biomedical technology for Respiration Analysis THrough optoelectronics). 


\section{References}

[1] Cluzel P, Similowski T, Chartrand-Lefebvre C, Zelter M, Derenne JP, Grenier P (2000) Diaphragm and chest wall: assessment of the inspiratory pump with MR Imaging - preliminary observations. Radiology 215:574-583.

[2] Gauthier AP, Verbanck S, Estenne M, Segebarth C, Macklem PT, Paiva M (1994) Three-dimensional reconstruction of in vivo human diaphragm shape at different lung volumes. J Appl Physiol 76:495-506.

[3] Gierada DS, Curtin JJ, Erickson SJ, Prost RW, Strandt JA, Goodman LR (1997) Fast gradient echo magnetic resonance imaging of the normal diaphragm. J Thorac Imaging $12: 70-74$

[4] Gierada DS, Hakimian S, Slone RM, Yusen RD (1998) MR Analysis of Lung Volume and Thoracic Dimensions in Patients with Emphysema Before and After Lung Volume Reduction Surgery. American Journal of Radiology 170:707-714.

[5] Paiva M, Verbanck S, Estenne M, Poncelet B, Segebarth C, Macklem PT (1992) Mechanical implications of in vivo human diaphragm shape. J Appl Physiol 72:14071412.

[6] Pettiaux N, Cassart M, Paiva M, Estenne M (1997) Three-dimensional reconstruction of human diaphragm with the use of spiral computed tomography. J Appl Physiol 82:998-1002.

[7] Carter EA, Cleverley JR, Delany DJ, Lea RE (1996) Cine MRI in the diagnosis of a ruptured right hemidiaphragm. Clin Radiol 51:137-140. 
[8] Gierada DS, Curtin JJ, Erickson SJ, Prost RW, Strandt JA, Goodman LR (1995)

Diaphragmatic motion: fast gradient-recalled-echo MR Imaging in healthy subjects. Radiology 194:879-884.

[9] Kanematsu M, Takeyoshi I, Ryozo M, Yoshiharu Y, Hiroo G, Yasuhiro S, Hidetaka D. (1995) Dynamic MRI of the diaphragm. J Comput Assist Tomogr 19:67-72.

[10] Sakai S, Murayama S, Hashiguchi N, Masuda K (1997) Bronchogenic carcinoma invasion of the chest wall: evaluation with dynamic cine MRI during breathing. $\mathrm{J}$ Comput Assist Tomogr 21:595-600.

[11] Cassart M, Pettiaux N, Genevois PA, Paiva M, Estenne M (1997) Effect of chronic hyperinflation on diaphragm length and surface area. Am J Respir Crit Care Med 156:504-508.

[12] Promayon E, Baconnier P, Puech C (1997) Physically-based model for simulating the human trunk respiration movements. In: Troccaz J, Grimson E, Mösges R (ed) Lecture notes in Computer Scienc. Springer-Verlag, Berlin Heidelberg, 1205:379-388.

[13] Carnevali P, Ferrigno G, Aliverti A, Pedotti A (1996) A new method for 3D optical analysis of chest wall motion. Technol Health Care 4:43-65. 


\section{Table}

\begin{tabular}{|l|c|}
\hline \multicolumn{1}{|c|}{ Plane } & Mean Excursion (cm) \\
\hline Anterior & 1.64 \\
Right dome & 2.95 \\
Left dome & 4.12 \\
Posterior & 4.14 \\
\hline
\end{tabular}

Table 1. Mean excursion for maximal amplitude breathing of the observed nodes located on four coronal planes: anterior plane, RA plane, LA plane, and posterior plane. 


\section{Captions for illustrations}

Figure 1. (a) MRI series and respiratory signal (from a BIOPAC MP100 system) were acquired at the same time, during both types of respiration, example is give for the quiet breathing maneuver. (b) Slices are numbered using their position in the volume from 1 to 15 . The example, is given for the sagittal slices numbering. Image acquisition times are marked by . A 3D reconstructed phase is composed by gathering 15 sagittal images and 15 coronal images taken during the same respiratory phase. For instance in (c), to reconstruct the 3D-RP of phase 1, slices 1 and 2 can be taken from cycle 1, slice 6 from cycle 12 , and slice 5 can either be taken from cycle 1 or from cycle 12 , and so forth until the volume is completed.

Figure 2. Our software was able to display the MR image volume in a 3D interactive environment on a PC. The initial 3D flat surface mesh nodes are visible on the bottom (in yellow). The radiologist can directly segment the diaphragm by interactively deforming the initial surface mesh in order to fit the diaphragmatic contour (resulting mesh is in red). A node is being moved on the selected slice (yellow node); other slices of the volume are represented by their frames (in white).

Figure 3. 3D reconstructed diaphragmatic surfaces during a respiratory cycle (phases to ), from the beginning of the inspiration (upper left, phase ), to the end of expiration (lower middle, phase ). A posterior view of the diaphragm at phase shows its lumbar attachments (lower right).

Figure 4. The final surface mesh was composed of 289 nodes. A 3D surface mesh described a 3D surface composed by 1514 triangles. This 3D reconstructed diaphragmatic surface corresponds to the beginning of the inspiration (phase 1). The "volume under diaphragm" is represented.

Figure 5. Craniocaudal displacement of the diaphragm in the anterior plane, in the right and left apex planes, and in the posterior plane during "maximal amplitude breathing". The posterior coronal plane includes 2 fixed median nodes corresponding to costovertebral attachments of diaphragmatic crus. The beginning of inspiration is plotted in black, and the end in gray.

Figure 6. Diaphragmatic areas in $\mathrm{cm} 2$ from the beginning of inspiration (phase 1) to the end of expiration (phase 10) during "quiet breathing" (black) and "maximal amplitude breathing" (gray).

Figure 7. "Volume under diaphragm" absolute variations in $\mathrm{cm} 3$ from the beginning of inspiration (phase 1) to the end of expiration (phase 10) during "quiet breathing" (black) and "maximal amplitude breathing" (gray).

Figure 8. Surface/Volume ratio during "quiet breathing" (black) and "maximal amplitude breathing" (gray). 


\section{Illustrations}
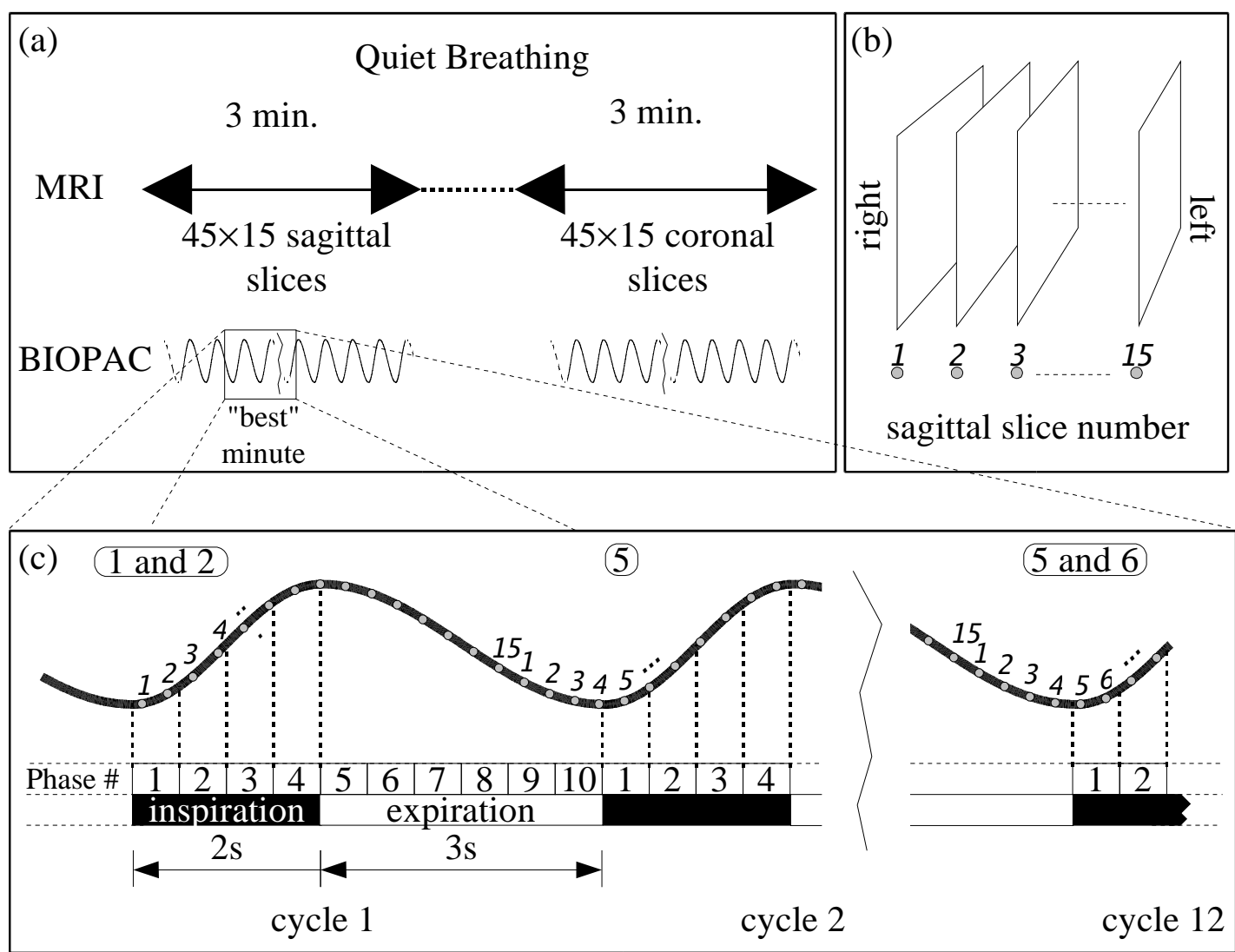

Figure 1. (a) MRI series and respiratory signal (from a BIOPAC MP100 system) were acquired at the same time, during both types of respiration, example is give for the quiet breathing maneuver.

(b) Slices are numbered using their position in the volume from 1 to 15 . The example, is given for the sagittal slices numbering. Image acquisition times are marked by ${ }^{\circ}$.

A $3 \mathrm{D}$ reconstructed phase is composed by gathering 15 sagittal images and 15 coronal images taken during the same respiratory phase.

For instance in (c), to reconstruct the 3D-RP of phase 1, slices 1 and 2 can be taken from cycle 1 , slice 6 from cycle 12, and slice 5 can either be taken from cycle 1 or from cycle 12 , and so forth until the volume is completed. 


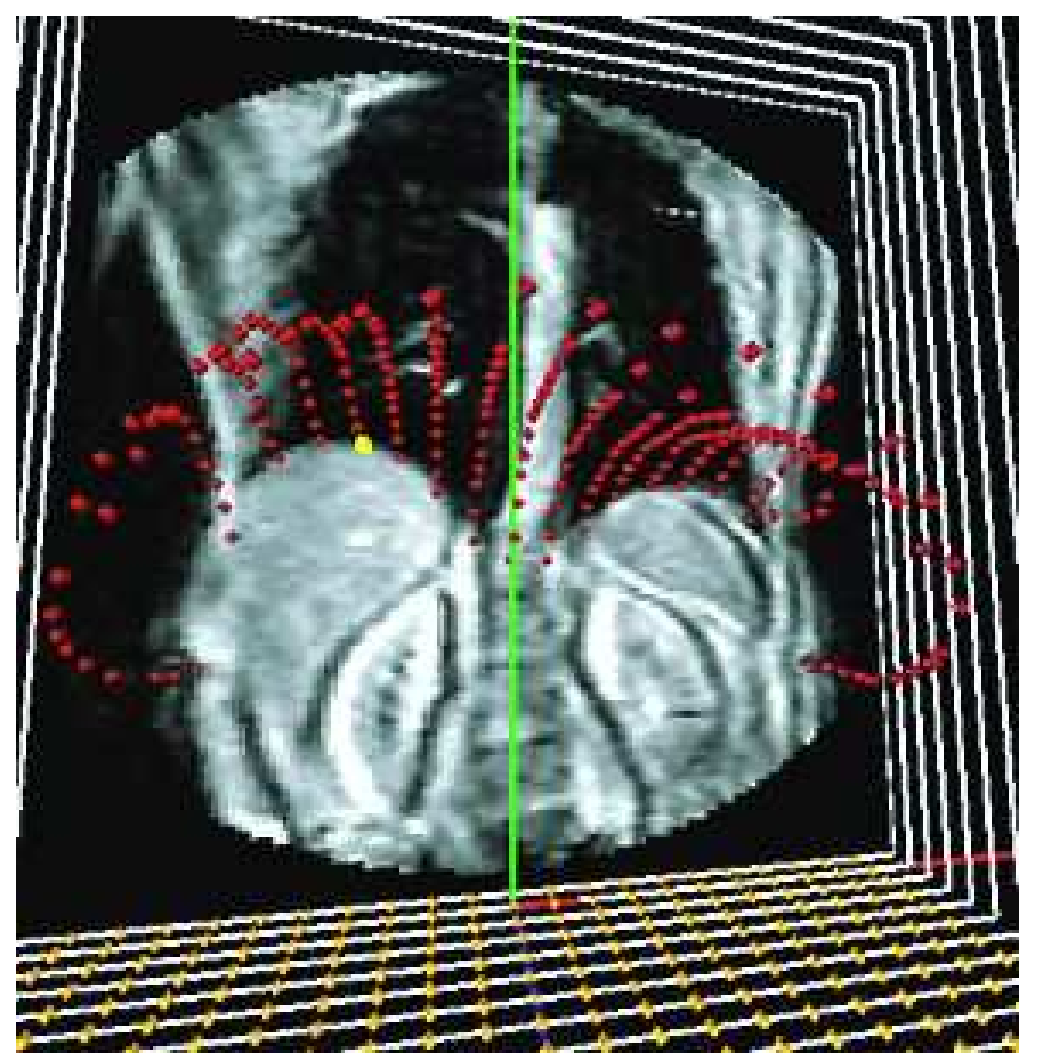

Figure 2. Our software was able to display the MR image volume in a $3 \mathrm{D}$ interactive environment on a PC. The initial 3D flat surface mesh nodes are visible on the bottom (in yellow). The radiologist can directly segment the diaphragm by interactively deforming the initial surface mesh in order to fit the diaphragmatic contour (resulting mesh is in red). A node is being moved on the selected slice (yellow node); other slices of the volume are represented by their frames (in white). 

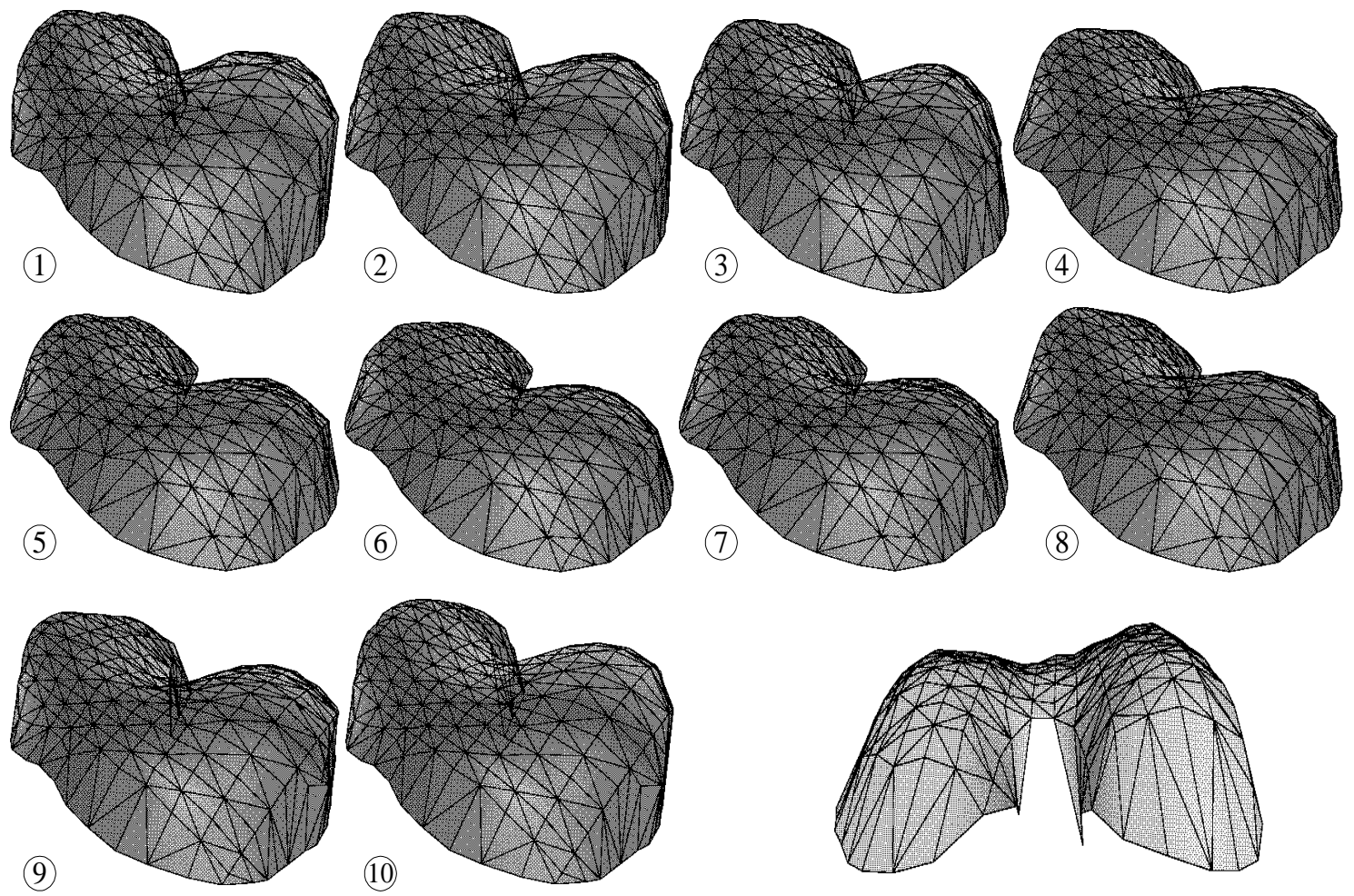

Figure 3. 3D reconstructed diaphragmatic surfaces during a respiratory cycle (phases (1) to (10), from the beginning of the inspiration (upper left, phase (1)), to the end of expiration (lower middle, phase (10)). A posterior view of the diaphragm at phase (6) shows its lumbar attachments (lower right). 


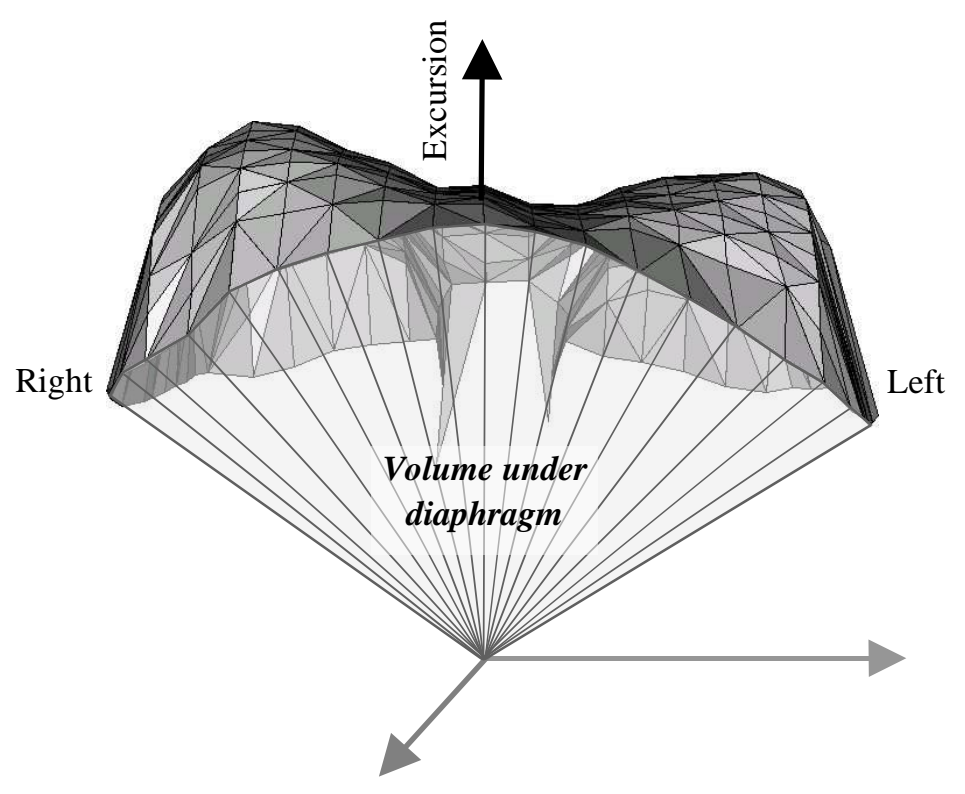

Figure 4. The final surface mesh was composed of 289 nodes. A 3D surface mesh described a 3D surface composed by 1514 triangles. This 3D reconstructed diaphragmatic surface corresponds to the beginning of the inspiration (phase 1). The "volume under diaphragm" is represented. 


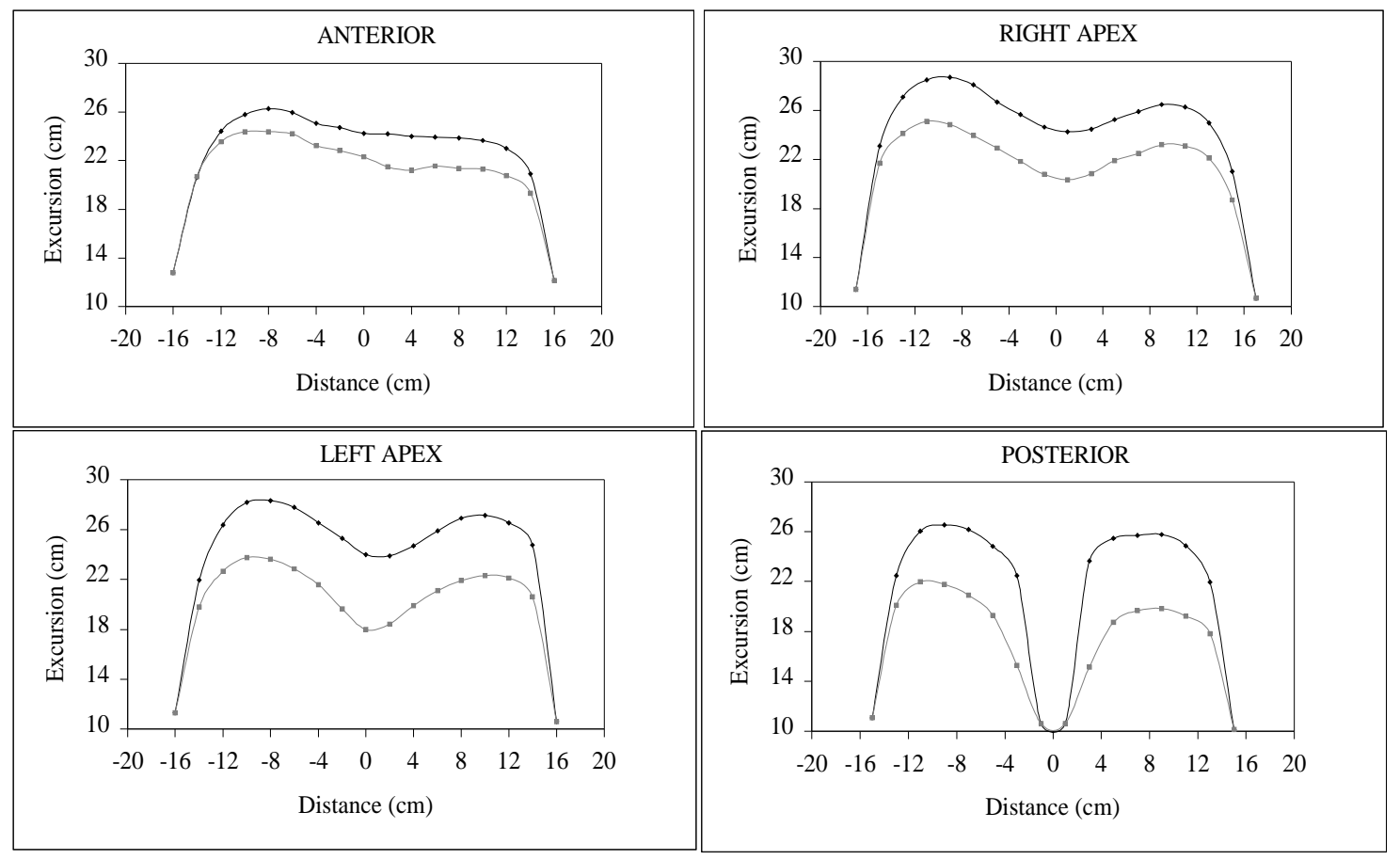

Figure 5. Craniocaudal displacement of the diaphragm in the anterior plane, in the right and left apex planes, and in the posterior plane during "maximal amplitude breathing". The posterior coronal plane includes 2 fixed median nodes corresponding to costovertebral attachments of diaphragmatic crus. The beginning of inspiration is plotted in black, and the end in gray. 


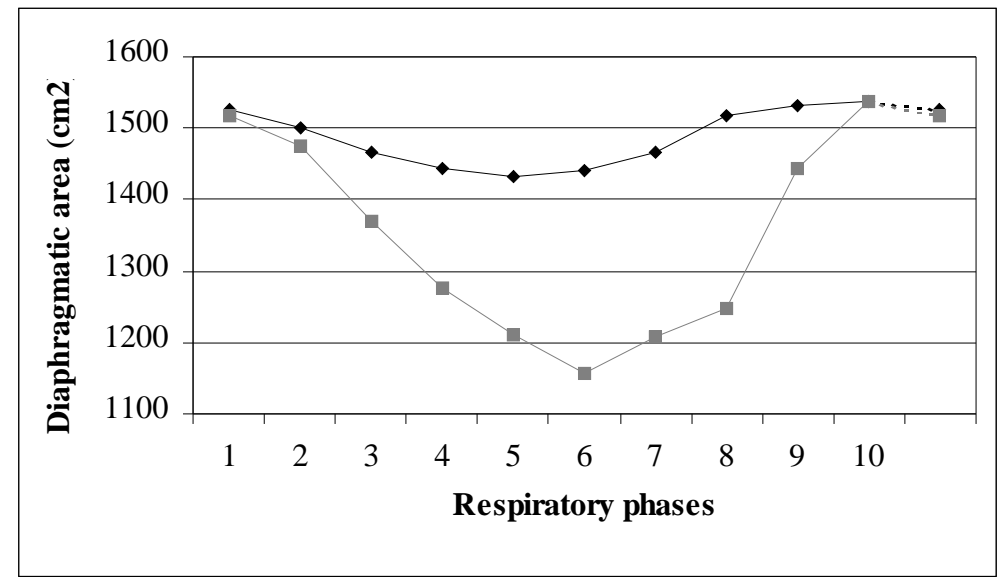

Figure 6. Diaphragmatic areas in $\mathrm{cm}^{2}$ from the beginning of inspiration (phase 1) to the end of expiration (phase 10) during "quiet breathing" (black) and "maximal amplitude breathing" (gray). 


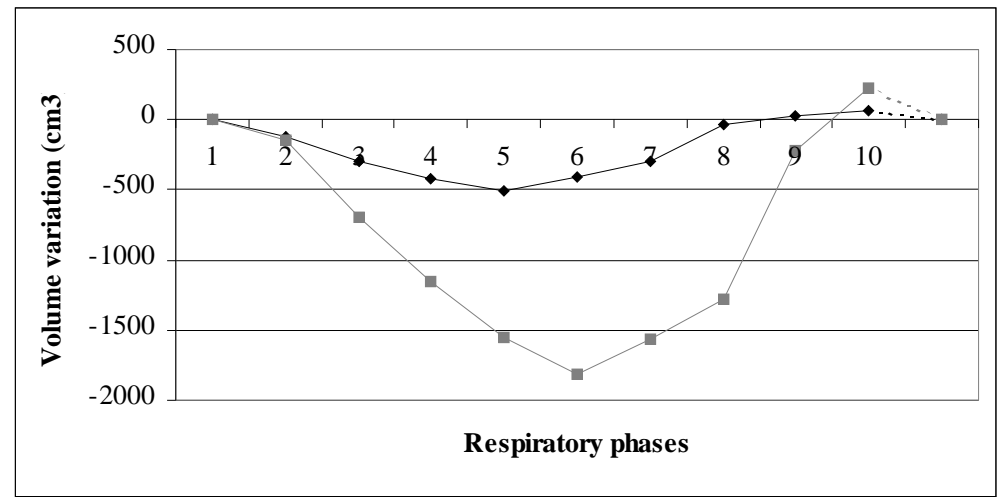

Figure 7. "Volume under diaphragm" absolute variations in $\mathrm{cm}^{3}$ from the beginning of inspiration (phase 1) to the end of expiration (phase 10) during "quiet breathing" (black) and "maximal amplitude breathing" (gray). 


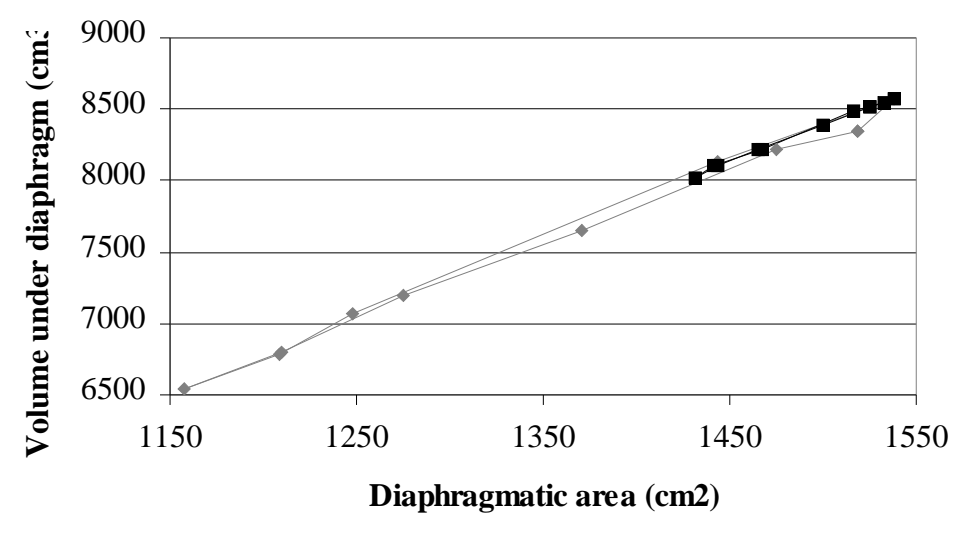

Figure 8. Surface/Volume ratio during "quiet breathing" (black) and "maximal amplitude breathing" (gray). 